\title{
On the Raman Instability in Degenerate Relativistic Plasmas
}

\author{
G.T.Chanturia ${ }^{1}$, V.I.Berezhiani ${ }^{1,2}$, S.M.Mahajan ${ }^{3}$ \\ 1 School of Physics, Free university of Tbilisi, Tbilisi 0159, Georgia \\ 2 Andronikashvili Institute of Physics (TSU), Tbilisi 0177, Georgia and \\ ${ }^{3}$ Institute for Fusion Studies, The University of Texas at Austin, Austin, Texas 78712, USA \\ The stimulated Raman scattering instability in a fully degenerate electron plasma is stud- \\ ied applying relativistic hydrodynamic and Maxwell equations. We demonstrated that the \\ instability develops for weakly as well as strongly relativistic degenerate plasma. It is shown \\ that in the field of strong radiation relativistically degenerate plasma effectively responses \\ as in the case of weak degeneracy.
}


Astrophysical objects contain very intense radiation sources with their spectral content spanning from radio to $\gamma$-ray emission [1]. Most types of stars, interstellar gas, active galactic nuclei, distant quasars etc. are either comprised by significant fraction of plasma, or are immersed in a plasma environment [2]. The nature of Radiation emitted by such plasmas is usually interpreted in terms of bremsstrahlung and synchrotron mechanism. However, under certain conditions the collective parametric effects as, for instance, stimulated Raman scattering (SRS) instability could leave definite signature on the radiation spectra [3]. Within the framework of classical plasmas, the SRS has been studied in a variety of systems like the laser-driven inertial confinement fusion, particle acceleration and plasma heating, and for probing of the plasma parameters in laboratory conditions [4]-[10]. Linear and nonlinear stages of SRS instability have been investigated for cold as well as relativistic hot plasma for EM radiation of arbitrary intensity. Possible role of SRS instability in astrophysical plasma is discussed in [11],[12] and references therein; it is shown that induced scattering could significantly affect radiation from sources with high brightness temperatures.

For the extremely high density plasmas pertinent to astrophysical objects such as in the interior of white dwarfs, the neutron or the pre-supernova stars and presumably, at the source of gamma ray bursts [2], the classical approximation breaks down; the relevant plasma is degenerate requiring Fermi-Dirac statistics for a correct description. The Fermi energy of the degenerate electron gas is greater than the binding energy with atomic nucleus and as a consequence all atoms are in an ionized state. The plasma number density is believed to be in the range from $N_{0}=10^{26} \mathrm{~cm}^{-3}$ to $N_{0}=10^{34} \mathrm{~cm}^{-3}$ and the matter behaves as a weakly coupled degenerate plasma provided that averaged interparticle distance is smaller than the thermal de Broglie wavelength [13]. At such densities, the degenerate electron gas must be treated relativistically even when its "temperature" is nonrelativistic, in fact, even zero.

The Fermi energy associated with a typical particles in a degenerate electron gas, $\epsilon_{F}=$ $m_{e} c^{2}\left(\gamma_{F}-1\right)\left(\right.$ the Fermi $\left.\gamma_{F}=\sqrt{1+p_{F}^{2} / m_{e}^{2} c^{2}}, p_{F}=m_{e} c\left(n_{R} / n_{c}\right)^{1 / 3}[14]\right)$, exceeds the rest mass energy for densities $n_{R}$ greater than the critical density $n_{c}=m_{e}^{3} c^{3} / 3 \pi^{2} \hbar^{3}=5.9 \times 10^{29} \mathrm{~cm}^{-3}$.

In studies of nonlinear self-interactions of high frequency EM and plasma waves in relativistic degenerate electron (as well as electron-positron) plasmas [15]-[18], it has been shown such plasmas can support stable localized EM structures for arbitrary level of degeneracy. The dynamics of these nonlinear EM structures could provides a theoretical basis for establishing the nature of the observed radiation. To the best of our knowledge, the SRS instability of EM radiation in degenerate relativistic plasma is not addressed so far. The SRS instability is a nonlinear parametric process when a powerful electromagnetic (EM) wave decays into a plasma wave and an EM wave. In an 
underdense plasma with $\omega_{0}>2 \omega_{e 0}$, where $\omega_{0}$ is the mean frequency of carrier EM pulses and $\omega_{e 0}=\left(4 \pi e^{2} N_{0} / m_{e}\right)^{1 / 2}$ is the electron plasma frequency, the SRS results in two EM sidebands upshifted and downshifted by the plasma frequency. For a relativistic degenerate electron gas, the radiation frequency at which SRS could be relevant, falls into soft or even hard $X$-ray band. Consequently, it could leave definite footprints in the spectra of astrophysical $X$-ray sources.

Our study of the SRS instability is based on the Maxwell equations and a relativistic electron plasma fluid model. We will work In terms of the familiar vectorial form of the fully covariant equations (see, for example [19]-[21]) that translate as (in the Coulomb gauge $\nabla \cdot \mathbf{A}=0$ )

$$
\begin{gathered}
\frac{\partial^{2} \mathbf{A}}{\partial t^{2}}-c^{2} \Delta \mathbf{A}+c \frac{\partial}{\partial t}(\nabla \varphi)-4 \pi c \mathbf{J}=0 \\
\Delta \varphi=4 \pi e\left(n_{R} \gamma-N_{0}\right)
\end{gathered}
$$

where the vector potential $\mathbf{A}$ and the scalar potential $\varphi$ are the appropriate components of the EM four vector $A^{\mu}=[\varphi, \mathbf{A}]$, and $\mathbf{J}=-e n_{R} \mathbf{p} / m_{e}$, and $-e c \gamma n_{R}$ constitute the electron four current $J^{\mu}$.

The ions just provide a neutralizing background, and have a density $N_{0}$ in their rest frame which is also taken to be the fiduciary/laboratory frame. The "rest" frame electron density, $n_{R}$, is a Lorentz scalar and is related to the laboratory frame density $N$ by the relation $N=\gamma n_{R}$. The relativistic $\gamma=\sqrt{1+\mathbf{p}^{2} / m_{e}^{2} c^{2}}$ is determined from the vector part $\mathbf{p}$ of the four momentum.

In the plasma with zero generalized vorticity $\boldsymbol{\Omega}=\nabla \times(G \mathbf{p}-e \mathbf{A} / c)=0$ the fluid equations for the electrons can be written as (see [15] for details):

$$
\begin{gathered}
\frac{\partial}{\partial t}\left(G \mathbf{p}-\frac{e}{c} \mathbf{A}\right)+\nabla\left(m_{e} c^{2} G \gamma-e \varphi\right)=0 \\
\frac{\partial}{\partial t} n_{R} \gamma+\nabla \cdot\left(n_{R} \mathbf{p} / m_{e}\right)=0
\end{gathered}
$$

In Eq.(3), the "thermal" mass enhancement factor $G$ is defined as $G=w /\left(n_{R} m_{e} c^{2}\right)$, where $w$ is the enthalpy per unit volume. The effective mass factor is, generally, a non-trivial function of plasma temperature and density for both classical Maxwell-Juttner, or the quantum Fermi-Juttner statistics. However, if the thermal energy of the plasma electrons is much lower than the Fermi energy, the plasma may be treated as cold, (i.e. having zero temperature). The zero temperature approximation will be adequate even for temperatures of order $10^{9} \mathrm{~K}$ allowing a particularly simple expression, $G=\gamma_{F}=\sqrt{1+\left(n_{R} / n_{c}\right)^{2 / 3}}[2]$. 
For an EM wave propagating in the $z$ direction(all dynamic variables vary only in $z$ and time $t)$, the vector potential has just transverse components $\mathbf{A}=\left(\mathbf{A}_{\perp}, 0\right)$. For this simplified 1-D propagation, Eq.(3) can be readily integrated to yield

$$
\mathbf{p}_{\perp}=\frac{\mathbf{A}_{\perp}}{\gamma_{F}}
$$

where

$$
\gamma_{F}=\sqrt{1+\left(R_{0} n\right)^{2 / 3}}
$$

and

$$
\gamma=\sqrt{1+\frac{\mathbf{A}_{\perp}^{2}}{\gamma_{F}^{2}}+p_{z}^{2}}
$$

in terms of the normalized variables $\mathbf{A}_{\perp}=\left(e \mathbf{A}_{\perp} / m_{e} c^{2}\right), \varphi=\left(e \varphi / m_{e} c^{2}\right), \mathbf{p}=(\mathbf{p} / m c)$ and $n=\left(n_{R} / N_{0}\right)$.

The parameter $R_{0}=\left(N_{0} / n_{c}\right)$ measures the strength of plasma degeneracy: for $R_{0}<<1$ the plasma is nonrelativistic, while for $R_{0} \geq 1$ plasma is in the relativistic degenerate state. Note that the dimensionless Fermi momentum now reads as $p_{F}=\left(R_{0} n\right)^{1 / 3}$.

The Maxwell Eq.(1) is reduced to the wave equation

$$
\frac{\partial^{2} \mathbf{A}_{\perp}}{\partial t^{2}}-c^{2} \frac{\partial^{2} \mathbf{A}_{\perp}}{\partial z^{2}}+\Omega_{e}^{2} \mathbf{A}_{\perp}=0
$$

where $\Omega_{e}=\left(4 \pi e^{2} n_{R} / m_{e} \gamma_{F}\right)^{1 / 2}$ is the frame independent plasma frequency, and is a Lorentz scalar. In the standard literature, it is conventional to define the frequency $\omega_{e 0}=\left(4 \pi e^{2} N_{0} / m_{e}\right)^{1 / 2}$ corresponding to the density in the lab. frame; the latter is related to the frame- invariant frequency through $\Omega_{e}=$ $\omega_{e 0}\left(n / \gamma_{F}\right)^{1 / 2}$ i.e., the invariant frequency, in this case, is seen as the lab frame frequency reduced by the relativistic Fermi effects. There are lots of subtle considerations in creating a strictly Lorentz invariant theory and the reader is referred to [19]-[21] for a deeper discussion.

The system of equations describing the longitudinal motion of electron plasma is given by

$$
\begin{gathered}
\frac{\partial}{\partial t} \gamma_{F} p_{z}+c \frac{\partial}{\partial z}\left(\gamma \gamma_{F}-\varphi\right)=0 \\
\frac{\partial}{\partial t} \gamma n+c \frac{\partial}{\partial z}\left(n p_{z}\right)=0
\end{gathered}
$$




$$
\frac{\partial^{2} \varphi}{\partial z^{2}}=\frac{\omega_{e 0}^{2}}{c^{2}}(n \gamma-1)
$$

We next carry out a stability analysis for the circularly polarized EM waves. The monochromatic pump EM wave with frequency $\omega_{0}$ and wave number $k_{0}$, is described by

$$
\mathbf{A}_{\perp}=\frac{1}{2}(\widehat{\mathbf{x}}+i \widehat{\mathbf{y}}) A \exp \left(-i \omega_{0} t+i k_{0} z\right)+c . c .
$$

Here $\widehat{\mathbf{x}}$ and $\widehat{\mathbf{y}}$ are the unit vectors, $A=a \exp (i \psi)$ where $a$ and $\psi$ are real valued amplitude and phase. The unperturbed state of the plasma is characterized by a purely transverse EM mode with constant amplitude $a=a_{0}\left(p_{z}=0=\varphi\right)$ and a constant comoving density of electrons $n_{0}$. This density is related to the lab frame density by the relation $n_{0}=1 / \gamma_{0}$ (in unites $n_{0}=N_{0} / \gamma_{0}$ ) where $\gamma_{0}=\sqrt{1+a_{0}^{2} / \gamma_{F 0}^{2}}$ and $\gamma_{F 0}=\sqrt{1+\left(R_{0} / \gamma_{0}\right)^{2 / 3}}$.

The dispersion relation that follows from Eqs.(8)-(12) reads

$$
\omega_{0}^{2}=k_{0}^{2} c^{2}+\Omega_{e 0}^{2}
$$

where $\Omega_{e 0}=\omega_{e 0}\left(n_{0} / \gamma_{F 0}\right)^{1 / 2}=\omega_{e 0} / \gamma_{m}^{1 / 2}$ is the relativistically modified electron plasma frequency and $\gamma_{m}=\gamma_{0} \gamma_{F 0}$. The expression for $\gamma_{m}$ can be written as $\gamma_{m}=\sqrt{1+p_{F 0}^{2}+a_{0}^{2}}$ where $p_{F 0}=$ $\left(R_{0} / \gamma_{0}\right)^{1 / 3}$ is the Fermi momentum. In the weakly degenerate case when $R_{0} \ll 1$ and $p_{F 0} \ll a_{0}$ the Eq. (13) coincides with the dispersion relation for cold classical plasma with $\Omega_{e 0}=\omega_{e 0} /\left(1+a_{0}^{2}\right)^{1 / 2}$, while for the arbitrary $R_{0}$ and in absence of pump wave $\left(a_{0}=0\right)$ the modified plasma frequency reads $\Omega_{e 0}=\omega_{e 0} /\left(1+R_{0}^{2 / 3}\right)^{1 / 2}$.

Most spectacular manifestations of plasma degeneracy, however, occur for radiation with relativistically large amplitudes, i.e., when $a_{0}>>1$, and the radiative modification of the effective electron- mass becomes comparable to or dominant over the degeneracy modification (see [20]) for a detailed discussion on radiative renormalization of the electron mass). For extreme relativistic amplitudes, the effective $\gamma$ simplifies to $\gamma_{m} \simeq \sqrt{1+a_{0}^{2}}$, essentially the expression for a non degenerate plasma. For arbitrary values of $a_{0}$ and $R_{0}$ (measuring the degeneracy strength), the relativistic factor $\gamma_{0}$ can be found from the following implicit relation:

$$
\left(\gamma_{0}^{2}-1\right)\left(1+\left(\frac{R_{0}}{\gamma_{0}}\right)^{2 / 3}\right)=a_{0}^{2}
$$


that can be readily solved. In Fig.1, we plot $\gamma_{0}$ vs $a_{0}$ for different values of $R_{0}$. Dashed part of each curve corresponds to $p_{F 0}<1$. For larger values of $R_{0}$, stronger fields are required to achieve large values of $\gamma_{0}$.

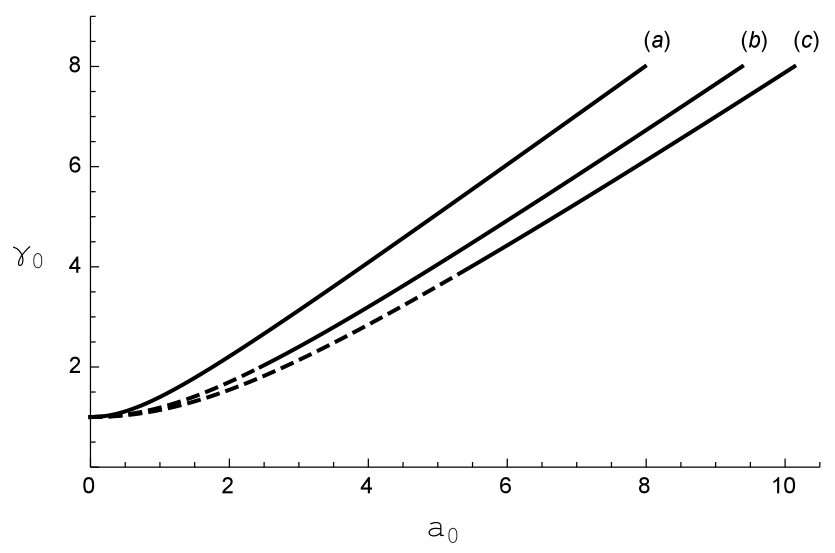

FIG. 1. Dependence of $\gamma_{0}$ on the EM field strength $a_{0}$ for different level of degeneracy: (a) $R_{0}=0.01$, (b) $R_{0}=2$, and (c) $R_{0}=4$.

A short message of the preceding results is that in the field of strong EM waves (see Fig.1), the effects of electron degeneracy become subdominant and the plasma responds as a weakly degenerate system.

To investigate the stability of ground wave solution we introduce the small perturbations in the system $f=f_{0}+\delta f\left(f_{0} \gg \delta f\right)$ where $f=\left(a, \psi, n, p_{z}, \varphi\right)$. Neglecting higher order terms, Eqs.(8) -(11) can be reduced to the following system of coupled equations:

$$
\begin{gathered}
\left(2 \omega_{0} \frac{\partial}{\partial t}+2 k_{0} c^{2} \frac{\partial}{\partial z}\right) \delta \psi+\left(\frac{\partial^{2}}{\partial t^{2}}-c^{2} \frac{\partial^{2}}{\partial z^{2}}\right) \frac{\delta a}{a_{0}}+ \\
+\Omega_{e 0}^{2}\left(\frac{\delta n}{n_{0}}-\frac{\delta \gamma_{F}}{\gamma_{F 0}}\right)=0 \\
\left(2 \omega_{0} \frac{\partial}{\partial t}+2 k_{0} c^{2} \frac{\partial}{\partial z}\right) \frac{\delta a}{a_{0}}+\left(c^{2} \frac{\partial^{2}}{\partial z^{2}}-\frac{\partial^{2}}{\partial t^{2}}\right) \delta \psi=0 \\
{\left[\frac{\partial^{2}}{\partial t^{2}}+\Omega_{e 0}^{2}\right]\left(\frac{\delta \gamma}{\gamma_{0}}+\frac{\delta n}{n_{0}}\right)=c^{2} \frac{\partial^{2}}{\partial z^{2}}\left(\frac{\delta \gamma}{\gamma_{0}}+\frac{\delta \gamma_{F}}{\gamma_{F_{0}}}\right)}
\end{gathered}
$$

while relations between $\delta \gamma$ and $\delta \gamma_{F}$ obtained from Eqs.(6)-(7) are 


$$
\begin{aligned}
\frac{\delta \gamma}{\gamma_{0}} & =\alpha\left(\frac{\delta a}{a_{0}}-\frac{\delta \gamma_{F}}{\gamma_{F_{0}}}\right) \\
\frac{\delta \gamma_{F}}{\gamma_{F_{0}}} & =\beta \frac{\delta n}{n_{0}}
\end{aligned}
$$

with $\alpha=\left(1-1 / \gamma_{0}^{2}\right)$ and $\beta=\left(1-1 / \gamma_{F_{0}}^{2}\right) / 3$.

To obtain the dispersion equation, we assume that all perturbations depend on the coordinates and time like $\delta f \sim \exp (-i \omega t+i k z)$. After some algebra the Eqs.(15)-(18) leads to dispersion relation

$$
\frac{\Omega_{e 0}^{2}}{2} A_{a} \frac{1}{D_{e}}\left(c^{2} k^{2}+\Omega_{e 0}^{2}-\omega^{2}\right)\left(\frac{1}{D_{-}}+\frac{1}{D_{+}}\right)=1
$$

where $D_{ \pm}=\left(\omega_{0} \pm \omega\right)^{2}-c^{2}\left(k_{0} \pm k\right)^{2}-\Omega_{e 0}^{2}$ and $D_{e}=\omega^{2}-\Omega_{e 0}^{2}-V_{m}^{2} k^{2} c^{2}$. Here $V_{m}^{2}=$ $\beta(1-\alpha) /(1-\alpha \beta), \quad A_{a}=\alpha(1-\beta) /(1-\alpha \beta)$ which can be presented in the explicit form as

$$
\begin{gathered}
V_{m}^{2}=\frac{1}{3} V_{F 0}^{2}\left(1-\frac{a_{0}^{2}}{\gamma_{m}^{2}}\right)\left(1-\frac{1}{3} V_{F 0}^{2} \frac{a_{0}^{2}}{\gamma_{m}^{2}}\right)^{-1} \\
A_{a}=\frac{a_{0}^{2}}{\gamma_{m}^{2} \gamma_{m 0}^{2}}
\end{gathered}
$$

where $V_{F 0}$ is the dimensionless $\left(V_{F 0} \rightarrow V_{F 0} / c\right)$ Fermi velocity defined by the relation $V_{F 0}=$ $\left(p_{F 0} / \gamma_{F 0}\right)$ and $\gamma_{m 0}=1 / \sqrt{1-V_{m}^{2}}$.

The dispersion relation (19) has the form similar to the one obtained in [8]. In this study the authors investigated the SRS in classical relativistic plasmas where thermal effects were included by a waterbag model for electron distribution. It so happens that the description of a fully degenerate plasma is formally analogous to a waterbag model [16] and this analogy is reflected in the form of Eq.(19). Important differences, induced by plasma degeneracy, are contained in the expressions (20)-(21) Note that in classical plasma $V_{m}$ is determined by the temperature (an independent parameter of the problem) while in a degenerate system, $V_{m}$ depends on plasma density ( determining the Fermi "temperature"). For ultra-relativistic degeneracy $\left(R_{0}>>1\right), V_{m} \rightarrow 1 / \sqrt{3}$. Hence, the maximal value of the parameter $\gamma_{m 0} \simeq 1.22$.

The solutions of equation (19) lead to resonance backward and forward SRS. Like in classical plasma, under certain simplified assumptions, temporal growth rates $(\Gamma=\operatorname{Im}(\omega))$ of instabilities 
can be obtained analytically. However, since the dispersion relation is a sixth order algebraic equation for $\omega$, numerical solutions may be more useful.

The transverse EM, and the plasma waves are coupled by the parameter $\chi=\Omega_{e 0}^{2} A_{a}$ and the growth rates of above mentioned instabilities are proportional to a certain power of the coupling parameter $\chi$. In weakly degenerate case $R_{0} \ll 1$ and finite strength of the field amplitude $\left(a_{0}\right)$ $\gamma_{m} \approx \sqrt{1+a_{0}^{2}}, V_{m} \ll 1$ and $\chi=\omega_{e 0}^{2} / \gamma_{m}^{3}$. In this case the dispersion relation (19) and, consequently, the results of instability coincide with the results obtained in classical cold plasma embedded in the field of arbitrary strong radiation [6], [7]. Here we would like to remark that our consideration is valid if the average kinetic energy of electrons $\left(\sim \epsilon_{F}\right)$ is larger than their interaction energy $\left(\sim e^{2} N_{0}^{1 / 3}\right)$. This condition is fulfilled for a sufficiently dense plasma when $N_{0} \gg 10^{23} \mathrm{~cm}^{-3}$ $\left(1 \gg R_{0} \gg 10^{-7}\right)$.

The main features and peculiarities of SRS instability in plasma with finite level of degeneracy parameter $R_{0}$ can be deduced by analyzing Eqs. (20)-(21) for different limiting cases. We look for the solution of Eq.(19) in the form $\omega=\omega_{L}+\delta \omega$, where $\omega_{L}=\sqrt{\Omega_{e 0}^{2}+V_{m}^{2} k^{2} c^{2}}$. The maximum growth rates are obtained when scattered wave is also resonant $D_{-}\left(\omega=\omega_{L}\right)=0$ leading to the following relation:

$$
\omega_{0}=\left(\Omega_{e 0}^{2}+c^{2} k^{2} V_{m}^{2}\right)^{1 / 2}+\left(\left(k_{0}-k\right)^{2}+\Omega_{e 0}^{2}\right)^{1 / 2}
$$

Using Eq.(22) one can show that in an underdense plasma $\left(\omega_{0}>2 \Omega_{e 0}\right)$ the wave vector of resonant modes $k$ lies in the range $0<k<2 k_{0}$. The modes with $k>k_{0}$ and $k<k_{0}$ lead respectively to backward and forward Raman instabilities. For highly transparent plasma $\left(\omega_{0} \gg \Omega_{e 0}\right)$ the backward Raman instability develops at $k \simeq 2 \omega_{0} / c\left(1+V_{m}\right)$ and $\omega_{L}=\left[\Omega_{e 0}^{2}+4 \omega_{0}^{2} V_{m}^{2} /\left(1+V_{m}\right)^{2}\right]^{1 / 2}$ [8]. Neglecting the nonresonant term $\left(\sim 1 / D_{+}\right)$in Eq.(19) and making approximations $D_{-}=$ $-2\left(\omega_{0}-\omega_{L}\right) \delta \omega$ and $\omega_{L}>>\delta \omega$ (and recalling that $\Omega_{e 0}=\omega_{e 0} / \gamma_{m}^{1 / 2}$ ) for the growth rate we get the following expression:

$$
\Gamma=\frac{\omega_{e 0}}{\sqrt{2}} \frac{a_{0}}{\gamma_{m}^{3 / 2}} \frac{\omega_{0}\left(1-V_{m}\right)}{\sqrt{\left(\omega_{0}-\omega_{L}\right) \omega_{L}}}
$$

For forward Raman scattering instability $k \ll k_{0} \approx \omega_{0} / c$ both downshifted and upshifted scattered waves are resonant modes $D_{ \pm}=2\left(\omega_{L} \pm \omega_{0}\right) \delta \omega$. The maximum growth rate,

$$
\Gamma=\frac{\omega_{e 0}^{2}}{2 \omega_{0}} \frac{a_{0}}{\gamma_{m}^{2} \gamma_{m 0}}
$$


occurs at $k=\Omega_{e 0} \gamma_{m 0} / c$ and $\omega_{L}=\Omega_{e 0} \gamma_{m 0}$.

As per expectations, in the limit of nonrelativistic degeneracy $\left(R_{0} \ll 1\right)$, Eqs.(23)-(24) tend to the classical results for a cold plasma. For finite level of degeneracy parameter $R_{0}$, and nonrelativistic strengths of the field $\left(a_{0} \ll 1\right)$, the growth rates of forward Raman instability is reduced by factor $\left(1+R_{0}^{2 / 3}\right)$ in comparison to weakly degenerate case, while for backward instability the reduction factor turns out to be $\left(1+R_{0}^{2 / 3}\right)^{3 / 4}$.

Most interesting regime, explored in this note, is that of a relativistic degenerate plasma $\left(R_{0} \geq 1\right)$ embedded in the field of a relativistically strong EM wave $\left(a_{0} \gg 1\right)$. In the regime of very strong radiation $\left(a_{0} / \gamma_{0} \gg 1\right.$ (see Fig.1 and comments after Eq.(13)), even a highly degenerate plasma responds as a weakly degenerate one. For ultrarelativistic amplitudes, defined by $R_{0} / \gamma_{0}<1$, the Fermi velocity becomes small $\left(V_{m} \rightarrow 0\right)$, and $\gamma_{m} \approx \sqrt{1+a_{0}^{2}}$. Consequently, the character of the SRS instability, in particular its growth rate, tends to approach the cold classical plasma results.

In this letter, we investigated the linear stage of SRS instability of a propagating arbitrary amplitude circularly polarized EM wave in a degenerate electron plasma. For 1-D wave propagation, it is demonstrated that in the field of ultra-relativistic amplitude waves, the relativistic degenerate plasma effectively responds as a weakly degenerate plasma due to reduction of Fermi momentum. While for weak, nonrelativistic amplitudes (but relativistic degenerate plasma) the growth rates of instability reduce with increasing density.

The elucidation of the SRS instability induced by ultra strong EM waves in a relativistic degenerate plasma is highly pertinent to understanding the dynamics of $X$-ray pulses emanating from compact astrophysical objects. It will be equally relevant for an exploration of nonlinear interactions between intense laser pulses and a dense degenerate plasma; the latter class of physical systems are likely to be realized in the next-generation intense laser solid density plasma experiments.

The research was supported by the Shota Rustaveli National Science Foundation grant (DI2016-14). The research of GT was supported by the Knowledge Foundation at the Free University of Tbilisi.

1 M. C. Begelman, R. D. Blandford, and M. D. Rees, Rev. Mod. Phys. 56, 255 (1984).

2 S.L. Shapiro and S.A. Teukolsky, Black Holes, White Dwarfs, and Neutron Stars: The Physics of Compact Objects (Wiley-VCH, Weinheim, 2004). 
3 V.V.Zheleznyakov, Radiation in astrophysical plasmas (Kluwer Academic Publishers, 1996).

4 W. L. Kruer, The Physics of Laser Plasma Interactions (Addison-Wesley, New York, 1988).

5 C. J. McKinstrie and R. Bingham, Phys. Fluids B4, 2626 (1992).

6 C. D. Decker, W. B. Mori, K.C. Tzeng, and T. Katsouleas, Phys. Plasmas 3, 2047 (1996).

7 A. S. Sakharov and V. I. Kirsanov, Phys. Rev. E49, 3274 (1994).

8 Z.M. Sheng, K. Mima, Y.Sentoku, and K. Nishihara, Phys. Rev. E61, 4362 (2000).

9 Y. Zhao et al., Phys. Plasmas 21, 112114 (2014).

10 D. Kaganovich et al., Phys. Plasmas 23, 123104 (2016).

11 S.A. Kaplan and V.N. Tsytovich, Plasma Astrophysics (Pergamon Press, 1973).

12 Y. Lyubarsky, ApJ 682, 1443 (2008).

13 L.D. Landau and E.M. Lifshitz, Statistical Physics (Pergamon Press, 1980).

14 F. Haas, J. Plasma Phys. 82, 705820602 (2016).

15 G. Mikaberidze, and V.I. Berezhiani, Phys. Lett. A 379, 2730 (2015).

16 F. Haas, I. Kourakis, Plasma Phys.Control. Fusion 57, 044006 (2015).

17 V.I. Berezhiani and N.L. Shatashvili, Phys. Plasmas 23, 104502 (2016)

18 V.I. Berezhiani, N.L. Shatashvili, and N.L. Tsintsadze, Phys. Scr. 90, 068005 (2015).

19 S. M. Mahajan, Phys. Rev. Lett. 90, 035001 (2003).

20 S. M. Mahajan and F. A. Asenjo, Phys. Plasmas 23, 056301 (2016).

21 S. M. Mahajan and F. A. Asenjo, J. Plasma Phys. 83, 905830101 (2017). 